\title{
L-mimosine and hypoxia can increase angiogenin production in dental pulp- derived cells
}

\author{
Klara Janjic ${ }^{1,2}$, Michael Edelmayer ${ }^{2,3}$, Andreas Moritz $^{1,2}$ and Hermann Agis ${ }^{1,2^{*}}$ (D)
}

\begin{abstract}
Background: Angiogenin is a key molecule in the healing process which has been successfully applied in the field of regenerative medicine. The role of angiogenin in dental pulp regeneration is unclear. Here we aimed to reveal the impact of the hypoxia mimetic agent L-mimosine (L-MIM) and hypoxia on angiogenin in the dental pulp.

Methods: Human dental pulp-derived cells (DPC) were cultured in monolayer and spheroid cultures and treated with L-MIM or hypoxia. In addition, tooth slice organ cultures were applied to mimic the pulp-dentin complex. We measured angiogenin mRNA and protein levels using QPCR and ELISA, respectively. Inhibitor studies with echinomycin were performed to reveal the role of hypoxia-inducible factor (HIF)-1 signaling.

Results: Both, L-MIM and hypoxia increased the production of angiogenin at the protein level in monolayer cultures of DPC, while the increase at the mRNA level did not reach the level of significance. The increase of angiogenin in response to treatment with L-MIM or hypoxia was reduced by echinomycin. In spheroid cultures, L-MIM increased angiogenin at protein levels while the effect of hypoxia was not significant. Angiogenin was also expressed and released in tooth slice organ cultures under normoxic and hypoxic conditions and in the presence of L-MIM.
\end{abstract}

Conclusions: L-MIM and hypoxia modulate production of angiogenin via HIF-1 differentially and the response depends on the culture model. Given the role of angiogenin in regeneration the here presented results are of high relevance for pre-conditioning approaches for cell therapy and tissue engineering in the field of regenerative endodontics.

Keywords: Angiogenin, ANG, Dental pulp, Prolyl hydroxylases, Prolyl hydroxylase inhibitors, Regeneration, Hypoxia, Hypoxia mimetic agents, Microtissues, Echinomycin

\section{Background}

Angiogenin (Angiogenin / ANGIOGENIN) is a key molecule in the healing process which has been successfully applied in the field of regenerative medicine and oncology [1-7]. The applications comprise the support of wound healing and bone regeneration where it has successfully stimulated angiogenesis and tissue regeneration in experimental settings $[3,4,8]$. While the known relevance of angiogenin is based on a broad spectrum of data in regenerative medicine, knowledge about the role

\footnotetext{
*Correspondence: hermann.agis@meduniwien.ac.at

'Department of Conservative Dentistry and Periodontology, School of

Dentistry, Medical University of Vienna, Sensengasse 2a, 1090 Vienna, Austria

${ }^{2}$ Austrian Cluster for Tissue Regeneration, Donaueschingenstr. 13, 1200

Vienna, Austria

Full list of author information is available at the end of the article
}

of angiogenin in regenerative endodontics and the dental pulp in general is currently limited. In vitro, cells of the dental pulp produce angiogenin / ANGIOGENIN and fast-setting calcium-silicate cements can modulate the production of angiogenin / ANGIOGENIN [9, 10]. In inflammation, innate immunity, neuroprotection, and tissue regeneration ANGIOGENIN represents a factor with high relevance also for regenerative endodontics [11-14].

ANGIOGENIN is a secreted protein and part of the ribonuclease superfamily for which reason it is also known as ribonuclease 5 [8]. The ANGIOGENIN encoding gene angiogenin is found on chromosome 14q11 [15]. The $14 \mathrm{kDa}$ basic single-chain protein consists of 
123 amino acids [8] and has 33\% sequence identity and $65 \%$ homology with bovine pancreatic RNase A as well as the same general catalytic residues [16]. The structure of the ANGIOGENIN protein is characterized by two $\alpha$ helices, seven $\beta$-sheets, and three disulfide bonds [8].

Angiogenin / ANGIOGENIN is found in the early phase during hard and soft tissue regeneration $[17,18]$. ANGIOGENIN is involved in angiogenesis and thus a key factor in cancer genesis but also tissue regeneration [1-7]. Various pro-angiogenic factors such as vascular endothelial growth factor, fibroblast growth factor, and endothelial growth factor stimulate angiogenin / ANGIOGENIN production $[8,19]$. In the complex process of angiogenesis, ANGIOGENIN induces a variety of cell responses. ANGIOGENIN binds to endothelial and smooth muscle cells triggering cell migration, cell invasion, proliferation of endothelial cells, and formation of tubular structures $[19,20]$. These functions are based on ANGIOGENIN's ribonuclease activity, basement membrane degradation, signaling transduction, and nuclear translocation [21, 22]. Furthermore, emerging evidence supports the notion that ANGIOGENIN is involved in the cell response to stress and cell survival [23]. All these processes highlight the relevance of ANGIOGENIN and led to the development of therapeutic strategies which target this mechanism [3,4].

Hypoxia-based strategies are a novel and promising approach to stimulate healing of the dental pulp in regenerative endodontics. While this approach emerged in a wave of various hypoxia-based approaches in regenerative medicine which dramatically improved our understanding of the effects of hypoxia, the impact of hypoxia in the dental pulp is not fully understood [24-30]. There is exisiting evidence from other fields of research that hypoxia can induce angiogenin / ANGIOGENIN via the transcription factor hypoxia-inducible factor (HIF)-1 [31, 32]. The role of ANGIOGENIN in the dental pulp is, however, unknown.

Here we hypothesized that hypoxia stimulates the production of angiogenin / ANGIOGENIN in the pulp in 2D monolayer, 3D spheroid, and tooth slice organ cultures. We show that the hypoxia mimetic agent Lmimosine (L-MIM) and hypoxia modulate production of angiogenin / ANGIOGENIN via HIF-1 differentially and the response depends on the culture model. These results are of high relevance for pre-conditioning approaches for cell therapy and tissue engineering in the field of regenerative endodontics which aim to improve the pro-angiogenic capacity of the transplanted cells.

\section{Methods}

\section{Cell culture}

Human dental pulp-derived cells (DPC) were prepared from extracted third molars after informed consent was given by the donors (Ethics Committee of the Medical University of Vienna, Vienna, Austria). Patients were recruited at the School of Dentistry, Medical University of Vienna, Vienna, Austria. The extraction of third molars from donors was part of standard care. The dental pulp was exposed and the tissue was collected. Explant cultures were done in $\alpha$-minimal essential medium $(\alpha-$ MEM) (Invitrogen Corporation, Carlsbad, CA, USA) with $10 \%$ fetal calf serum (FCS; PAA Laboratories, Linz, Upper Austria, Austria) and antibiotics at $37{ }^{\circ} \mathrm{C}, 5 \%$ $\mathrm{CO}_{2}$, and $95 \%$ atmospheric moisture.

\section{Monolayer culture of dental-pulp-derived cells}

For the monolayer cultures, DPC were seeded at 50,000 cells $/ \mathrm{cm}^{2}$ and incubated overnight. Then DPC were treated with $1 \mathrm{mM}$ L-MIM or hypoxia for $24 \mathrm{~h}$. This dose was based on the results of previous in vitro studies [24, 33]. For hypoxia, an established assay was applied with minor modifications [34]. In brief, DPC were placed into a BD GasPak EZ Pouch system for hypoxic conditions (Becton, Dickinson and Company, Franklin Lakes, NJ, USA). In indicated experiments, cells were also treated with echinomycin at $1 \mu \mathrm{M}$ to block HIF-1 activity. Untreated cells cultured under normoxic conditions served as control.

\section{Spheroid culture of dental pulp-derived cells}

For the spheroid cultures, 3D Petri Dishes ${ }^{\ominus}$ (Microtissues Inc., Providence, RI, USA) were used. The dishes were filled with $2 \%$ agarose to produce molds with 35 circular recesses. The molds were then soaked in $\alpha$-MEM supplemented with 10\% FCS and antibiotics (Invitrogen Corporation, Carlsbad, CA, USA). Afterwards, the molds were transferred to the well plates and $75 \mu \mathrm{L}$ of cell suspension with 7,300,000 cells $/ \mathrm{mL}$ were pipetted into the molds. After 10 min settling time the well was filled with cell culture medium as described in the manufacturer's description and incubated overnight. Then spheroids of DPC were stimulated with L-MIM or hypoxia as described above in the monolayer cultures.

\section{Tooth slice organ culture}

After informed consent was obtained, $600 \mu \mathrm{m}$ thick slices from extracted third molars without any sign of inflammation were prepared. For slicing the teeth, a diamond saw (Exakt $300 \mathrm{CL}$ and D64 0,2 mm, EXAKT Norderstedt, Germany) was used. Directly after cutting, the slices were placed in $\alpha$-MEM (Invitrogen Corporation) supplemented with $10 \%$ FCS and antibiotics for $48 \mathrm{~h}$ at $37{ }^{\circ} \mathrm{C}, 5 \% \mathrm{CO}_{2}$, and $95 \%$ atmospheric moisture. Then the tooth slices were treated with L-MIM at $1 \mathrm{mM}$ or hypoxia for $48 \mathrm{~h}$ according to the concentrations used in previous studies [24, 35]. From one set of tooth slices total RNA was extracted and angiogenin mRNA levels were assessed using RT-qPCR. Culture supernatants were assessed by ELISA to quantify 
the amount of ANGIOGENIN protein. Furthermore, MTT assays were applied on the tooth slices to quantify the vital tissue. In brief, tooth slices were incubated with 3-(4,5-dimethylthiazol-2-yl)-2,5-diphenyltetrazolium bromide (MTT) at $1 \mathrm{mg} / \mathrm{mL}$ for $2 \mathrm{~h}$. Formazan was solubilized with DMSO and measured with the Synergy HTX multiplate reader (BiotTek, Bad Friedrichshall, Germany) at $550 \mathrm{~nm}$. ANGIOGENIN levels in the culture medium were normalized to formazan formation of the pulp in the tooth slices.

\section{Reverse transcription quantitative polymerase chain reaction}

One day after treatment with L-MIM or hypoxia, total RNA was isolated from DPC of both culture models as well as the pulp tissue of the tooth slices using the RNeasy Plus Mini Kit, according to the protocol of the manufacturer (Qiagen, Hilden, NW, Germany). We performed synthesis of cDNA with the High Capacity cDNA Reverse Transcription Kit (Applied Biosystems, Carlsbad, CA) and diluted for reverse transcription quantitative polymerase chain reaction (RT-qPCR) as recommended by the manufacturer. cDNA was amplified with TaqMan ${ }^{\circ}$ Real-Time PCR Master Mix (Applied Biosystems) and $\mathrm{TaqMan}^{\circ}$ assays (Applied Biosystems) for angiogenin. Gapdh served as housekeeping gene (Table 1). mRNA levels were calculated by the $\Delta \Delta \mathrm{Ct}$ method, relative to the gapdh gene expression levels.

\section{Immunoassays}

Supernatants of the monolayer, spheroid, and tooth slice organ cultures were assessed using ELISA for ANGIOGENIN (human angiogenin DuoSet ${ }^{\circ}$ ELISA kit R\&D Systems Europe, Ltd. Abingdon, UK). The absorbance was measured at $450 \mathrm{~nm}$ (Correction at $540 \mathrm{~nm}$ ) in a Synergy HTX multiplate reader (BiotTek). The concentration of total ANGIOGENIN was calculated with the standard curve method following the protocol of the manufacturer.

\section{Statistical analysis}

Statistical analysis was performed with IBM SPSS Statistics Version 23 (IBM Corporation, Armonk, NY, USA), using the Kruskal-Wallis-test post hoc Mann-Whitneytest corrected for multiple testing by Bonferroni correction. The level of significance was set at $p<0.05$.

Table 1 All TaqMan ${ }^{\oplus}$ assays that were used for qPCR are listed in this table ( Thermo Fisher Scientific, MA, USA)

\begin{tabular}{lll}
\hline Gene symbol & Gene name & Assay ID \\
\hline Ang & angiogenin & Hs04195574_sH \\
Gapdh & glyceraldehyde-3-phosphate dehydrogenase & Hs02758991_g1 \\
\hline
\end{tabular}

\section{Results}

L-mimosine and hypoxia increase the production of angiogenin / ANGIOGENIN in monolayer cultures of dental pulp-derived cells

We measured the production of angiogenin at the mRNA level in response to L-MIM and hypoxia. Angiogenin was expressed in monolayer cultures of DPC under normoxic conditions. Modulation of angiogenin by both, L-MIM and hypoxia did not reach the level of significance $(p<0.05)$ in DPC monolayer cultures at the mRNA level (Fig. 1 a). At the protein level L-MIM and hypoxia increased ANGIOGENIN compared to the untreated cells $(p<0.05)$ as observed in the culture supernatants (Fig. 1 b). Our results show that hypoxia mimetic agents and hypoxia can increase the release of ANGIOGENIN in DPC cultured in monolayers.

\section{Echinomycin reduces the increase of angiogenin / ANGIOGENIN in response to L-mimosine and hypoxia} To assess the role of HIF- $1 \alpha$ in the underlying mechanism, inhibitor studies with echinomycin, an inhibitor of HIF-1 function, were performed. Echinomycin reduced mRNA production of angiogenin in the presence of LMIM $(p<0.05)$ (Fig. 2 a, b). Furthermore, our data show that echinomycin reduces the effect of L-MIM and hypoxia on ANGIOGENIN protein production in DPC monolayer cultures $(p<0.05)$ (Fig. 2 c, d). These data demonstrate that HIF-1 activity is required for the effect of hypoxia mimetic agents and hypoxia on ANGIOGENIN production in monolayer cultures of DPC.

L-mimosine, but not hypoxia, stimulates the production of angiogenin / ANGIOGENIN in spheroid cultures of dental pulp-derived cells

To mimic the 3D matrix of the dental pulp we used the spheroid culture model. In these cultures, the increase of angiogenin mRNA upon L-MIM treatment did not reach the level of significance $(p>0.05)$ (Fig. 3 a). At the protein level ANGIOGENIN was increased by L-MIM (Fig. 3 b). The effect of hypoxia in the spheroid culture model did not reach the level of significance at both the mRNA and the protein level (Fig. 3 a, b). These suggest that the effect of hypoxia mimetic agents and hypoxia on ANGIOGENIN production are less pronounced under $3 \mathrm{D}$ than under 2D conditions.

\section{Angiogenin / ANGIOGENIN is produced in tooth slice organ cultures}

Next we aimed to reveal the impact of hypoxia mimetic agents and hypoxia on the production of ANGIOGENIN in the dental pulp. Therefore, we applied the tooth slice organ culture model. Our results show that angiogenin mRNA was expressed in pulp tissue under normoxia, hypoxia, and in the presence of L-MIM (Fig. 4 a). We found a trend to an increase in angiogenin upon 

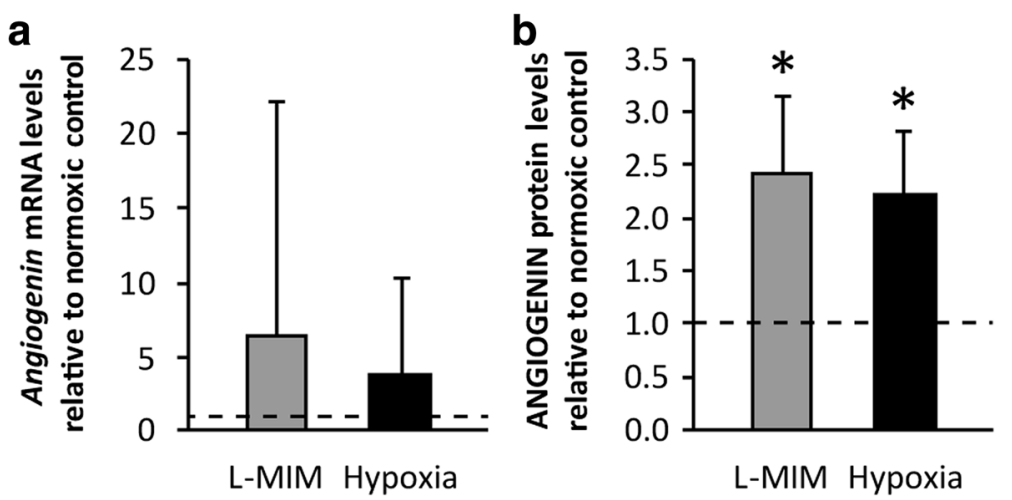

Fig. $1 \mathrm{~L}$-mimosine and hypoxia stimulate angiogenin / ANGIOGENIN production in monolayer cultures of dental pulp-derived cells. Dental pulp-derived cells (DPC) were incubated with L-mimosine (L-MIM) at $1 \mathrm{mM}$ or hypoxia for $24 \mathrm{~h}$ in monolayer cultures. mRNA of angiogenin (a) and protein of ANGIOGENIN (b) were assessed by RT-qPCR and ELISA, respectively. a Bars represent mRNA levels as mean + standard deviation, relative to the normoxic control (dashed line). Two independent experiments were performed with three donors. Kruskal Wallis test $p<0.05$. post hoc Mann-Whitney * $p<0.05 \mathbf{b}$ Bars show mean + standard deviation of ANGIOGENIN relative to the normoxic control (dashed line). Two independent experiments were performed with three donors. Kruskal Wallis test $p<0.05$. post hoc Mann-Whitney * $p<0.05$

treatment with L-MIM and hypoxia. Due to the limited number of tooth slices, no statistical evaluation of angiogenin mRNA was performed as the resulting power was too low. Interestingly, no trend for a modulation of ANGIOGENIN protein levels in the culture supernatant was observed upon treatment with L-MIM or hypoxia (Fig. 4 b). Again no statistical evaluation of ANGIOGENIN protein levels was performed as the resulting power was too low due to the limited number of tooth slices. Overall these data show that ANGIOGENIN is produced in the dental pulp, also in the presence of hypoxia mimetic agents and hypoxia.

\section{Discussion}

Angiogenin (angiogenin / ANGIOGENIN) is a key molecule in angiogenesis and healing. Here we show that
ANGIOGENIN is increased in monolayer cultures of DPC in response to treatment with the hypoxia mimetic agent L-MIM and hypoxia. This increase in ANGIOGENIN was abolished in the presence of the HIF-1 activity inhibitor echinomycin suggesting that active HIF-1 is required for the increase in ANGIOGENIN. ANGIOGENIN was also produced in 3D spheroid cultures and in tooth slice organ cultures. However, the response of the cultures to treatment with L-mimosine and hypoxia depends on the culture model.

Here we present novel evidence showing the regulation of ANGIOGENIN in DPC. The hypoxia mimetic agent L-MIM and hypoxic conditions stimulate ANGIOGENIN in monolayer cultures of DPC. These results are in line with previous studies on other cell lines of non-
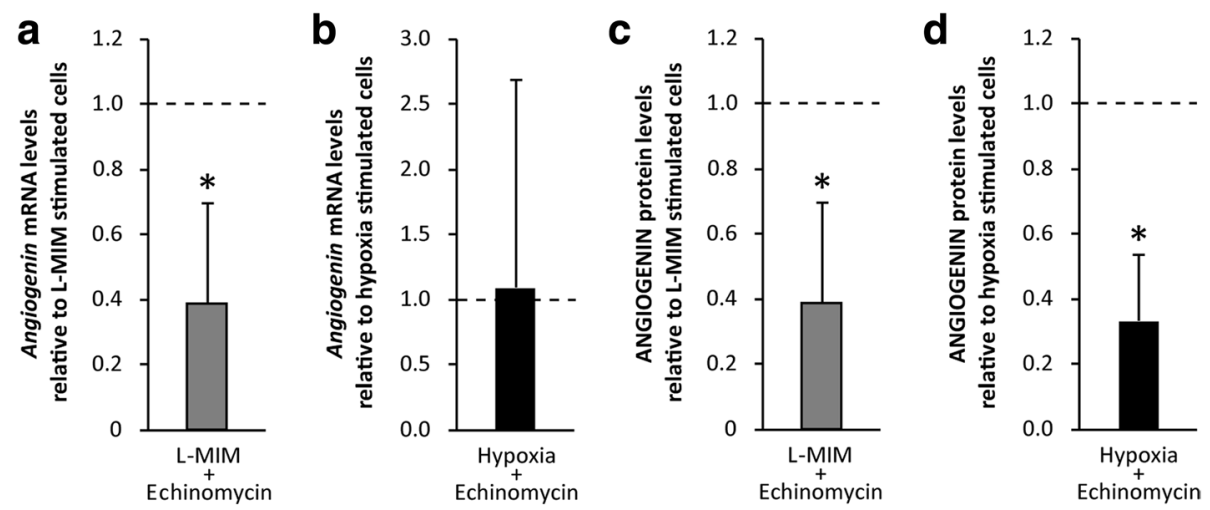

Fig. 2 Hypoxia inducible factor-1 signaling is involved in the increase of angiogenin / ANGIOGENIN upon stimulation with L-mimosine or hypoxia. Dental pulp-derived cells (DPC) in monolayer cultures were treated with L-mimosine (L-MIM) at $1 \mathrm{mM}$ or hypoxia with and without echinomycin at $1 \mu \mathrm{M}$ to for $24 \mathrm{~h}$. mRNA levels of angiogenin $(\mathbf{a}, \mathbf{b})$ and protein levels of ANGIOGENIN $(\mathbf{c}, \mathbf{d})$ were assessed by RT-qPCR and ELISA, respectively. $\mathbf{a}, \mathbf{b}$ Bars represent mRNA levels as mean + standard deviation, relative to the normoxic control (dashed line). Experiments were conducted twice with three different donors, respectively. Kruskal Wallis test $p<0.05$. post hoc Mann-Whitney ${ }^{*} p<0.05 \mathbf{c}$, $\mathbf{d}$ Bars represent mean + standard deviation of ANGIOGENIN relative to the normoxic control (dashed line). Experiments were conducted twice with 3 different donors, respectively. Kruskal Wallis test $p<0.05$. post hoc Mann-Whitney ${ }^{*} p<0.05$ 

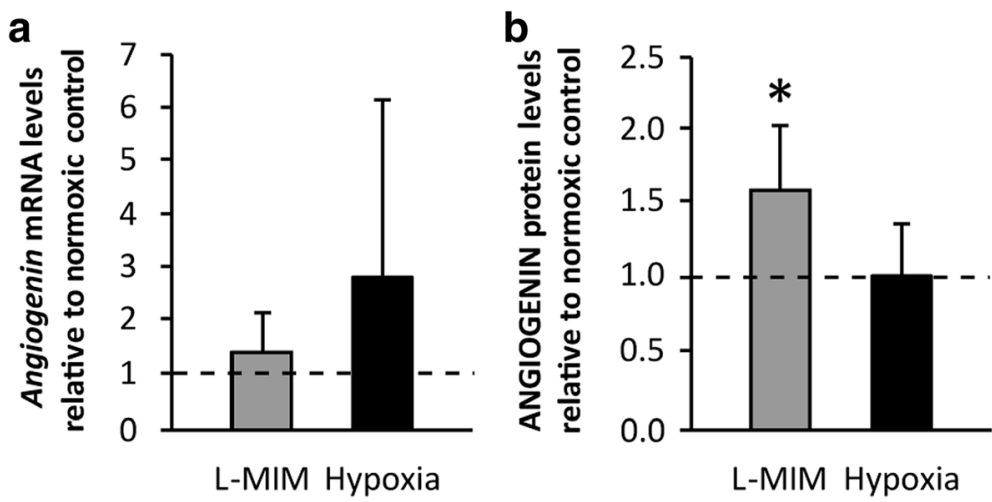

Fig. 3 Angiogenin / ANGIOGENIN production in spheroid cultures of dental pulp-derived cells in response to L-mimosine and hypoxia. Dental pulp-derived cells (DPC) in spheroid cultures were treated with L-mimosine (L-MIM) at $1 \mathrm{mM}$ or hypoxia for $24 \mathrm{~h}$. mRNA levels of angiogenin (a) and protein levels of ANGIOGENIN (b) were assessed by RT-qPCR and ELISA, respectively. a Bars represent mRNA levels as mean + standard deviation, relative to the normoxic control (dashed line). Experiments were conducted twice with 3 different donors, respectively. Kruskal Wallis test $p>0.05$. b Bars represent mean + standard deviation of ANGIOGENIN protein levels relative to the normoxic control (dashed line). Experiments were conducted twice with three different donors, respectively. Kruskal Wallis test $p<0.05$. post hoc Mann-Whitney * $p<0.05$ vs. control (dashed line)

oral origin such as retinal pigment endothelial cells or human renal proximal tubular epithelial cells in culture [31, 32].

Here we used DPC in monolayer cultures which represent a heterogeneous population and which can include cells, positive for mesenchymal stem cell markers, as well as low levels of hematopoetic stem cell markers [36-39]. In this setup it is unclear which population contributes to which extent to the observer effect. However, this setup closer represents the situation in the dental pulp than in isolated cell populations based on surface markers. To mimic the 3D structure of the pulp and therefore more closely the in vivo situation, we used $3 \mathrm{D}$ spheroid cultures of DPC in addition of 2D cultures [40]. Being aware of the background of a highly complex in vivo situation in the pulp-dentin complex which combines soft tissue containing fibroblasts, stem cells, blood vessels embedded in hard tissue, we therefore used the tooth slice organ culture [35]. Thereby we give a broad perspective on the effect of hypoxia mimetic agents and hypoxia on the production of angiogenin / ANGIOGENIN in the DPC. Our results show a differential response of angiogenin / ANGIOGENIN. It is therefore possible that cells cultured in the different 3D in vitro models are less sensitive to treatment with $\mathrm{L}$ mimosine or hypoxia than cells cultured in monolayer cultures. It is also possible that cells in the core of the spheroids and within the pulp tissue of the tooth slices have already reached low levels of oxygen. Further treatment with hypoxia or L-MIM might therefore not be as effective. However, comparing the response at mRNA levels with the response at protein levels in particular in
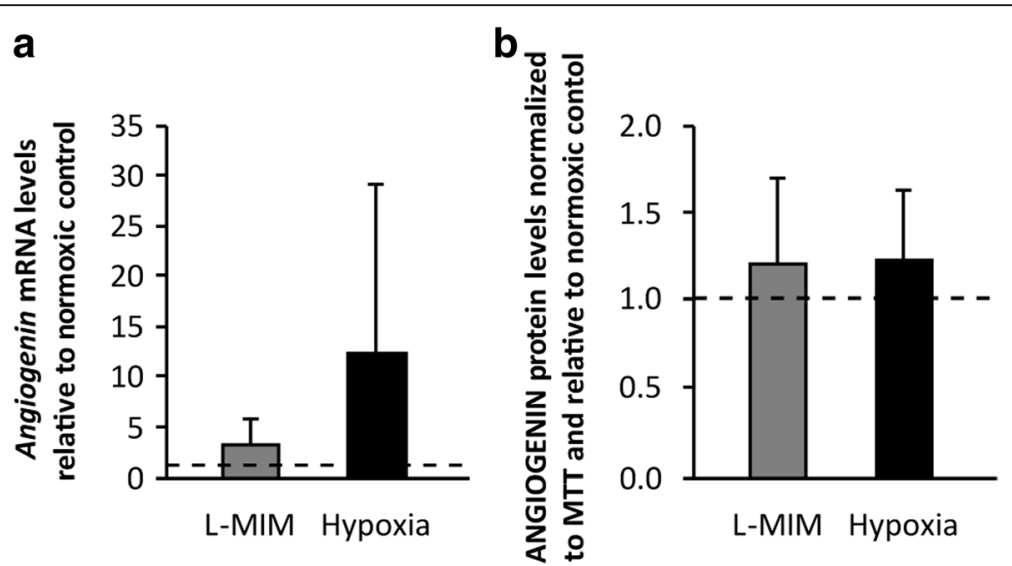

Fig. 4 Angiogenin / ANGIOGENIN is produced in tooth slice organ cultures. Tooth slices were cultured with and without L-mimosine (L-MIM) or hypoxia. mRNA levels of angiogenin (a) and protein levels of ANGIOGENIN (b) were assessed by RT-qPCR and ELISA, respectively. $\mathbf{a}$ Bars represent mRNA levels as mean + standard deviation, relative to the normoxic control from tooth slices of two donors. $\mathbf{b}$ Bars represent mean + standard deviation of ANGIOGENIN protein levels normalized to MTT and relative to the normoxic control (dashed line) from tooth slices of two donors 
the tooth slice model suggests that angiogenin / ANGIOGENIN is produced in response to L-MIM and hypoxia, but is not released in its full extent. A clear limitation of this study is that the timing for sample collection for the evaluation of mRNA and protein was limited to one time point. Based on previous studies a stimulation period of $24 \mathrm{~h}$ was chosen for monolayer and spheroid cultures and $48 \mathrm{~h}$ was chosen for tooth slice cultures which showed a hypoxia-induced cellular response $[24,33,35]$. Both 24 and $48 \mathrm{~h}$ have been shown to be within the time frame of the effect of hypoxia on angiogenin production in non-oral cells [41]. However, it might be possible that the peak of angiogenin expression and ANGIOGENIN protein production is not at the same time point. Thus the kinetics of angiogenin / ANGIOGENIN production remains to be determined in future studies.

Hypoxia-based strategies are a promising approach in regenerative endodontics. Several studies have shown that targeting the cellular oxygen sensors with hypoxia mimetic agents or by hypoxic conditioning can support the pro-angiogenic capacity of the cells and increase cell survival and grafting capacity [24-30]. Also cell-free approaches for regenerative medicine have been described using conditioned medium, also known as secretome of cells of the dental pulp for tissue regeneration [42, 43]. Hypoxic pre-conditioning can support the proangiogenic capacity [43-45]. Here, in the present study we are the first to present that hypoxia mimetic agents and hypoxia can stimulate the production of ANGIOGENIN in DPC and that the effect depends on the in vitro model. It is possible that the elevated ANGIOGENIN levels contribute to the high pro-angiogenic capacity of secretome for cells of the dental pulp. However, due to the low stability of angiogenin it is unclear to which extent ANGIOGENIN might play a role in the therapeutic application of the secretome. While also strategies with recombinant ANGIOGENIN have been reported, a key factor seems to be the controlled release of ANGIOGENIN at the defect site [3, 4]. This challenge has stimulated research in ANGIOGENIN releasing scaffolds in particular for bone regenerative applications. Another strategy is the gene therapy approach $[3,4,46]$. Adeno-associated virus-mediated angiogenin gene transfer showed promising results [46]. However, no angiogenin-based strategy for regenerative endodontics was reported yet and the role of angiogenin in the dental pulp remains an enigma. What we have contributed are insights into the way how hypoxia mimetic agents and hypoxia regulate the production of angiogenin.

\section{Conclusions}

In conclusion, our results show that the hypoxia mimetic agent L-MIM and hypoxia can stimulate angiogenin production in DPC and that this cellular response depends on HIF-1 activity. These results on the modulation of angiogenin in the dental pulp will be a primer for future studies which will address the role of angiogenin in pulp regeneration. Thus, the results of this study are of high relevance for pre-conditioning approaches in cell therapy and tissue engineering for regenerative endodontics.

\section{Abbreviations \\ ANG: Angiogenin; ANOVA: Analysis of variance; DPC: Dental pulp-derived cells; ELISA: Enzyme-linked immunosorbent assay; HIF: Hypoxia-inducible factor; L-MIM: L-mimosine; MTT: 3-(4,5-dimethylthiazol-2-yl)-2,5- diphenyltetrazolium bromide; APCR: Quantitative polymerase chain reaction; RT: Reverse transcription; VEGF: Vascular endothelial growth factor; a-MEM: a- minimal essential medium}

\section{Acknowledgements}

The authors thank Manuela Pensch for skillful technical assistance. The authors deny any conflict of interest. Our research was supported by the International Team for Implantology (ITI) Research Grant 1085_2015.

Funding

Our research was supported by the International Team for Implantology (ITI) Research Grant 1085_2015.

\section{Availability of data and materials \\ The datasets analyzed during the current study are available from the corresponding author on reasonable request.}

\section{Authors' contributions}

$\mathrm{KJ}$ was involved in study design, investigation, data analysis, and writing the manuscript. ME was involved in investigation, data analysis, and writing the manuscript. AM was involved in the study design, data interpretation, and manuscript preparation. HA was involved in study design, investigation, data analysis, writing, and submission of the manuscript. All authors read and approved the final manuscript.

\section{Competing interests}

The authors declare that they have no competing interests.

\section{Consent for publication}

Not applicable.

\section{Ethics approval and consent to participate}

The protocol was approved by the Ethics Committee of the Medical University Vienna (\#631/2007). Informed consent was given by the donors.

\section{Publisher's Note}

Springer Nature remains neutral with regard to jurisdictional claims in published maps and institutional affiliations.

\section{Author details}

${ }^{1}$ Department of Conservative Dentistry and Periodontology, School of Dentistry, Medical University of Vienna, Sensengasse 2a, 1090 Vienna, Austria. ${ }^{2}$ Austrian Cluster for Tissue Regeneration, Donaueschingenstr. 13, 1200 Vienna, Austria. ${ }^{3}$ Department of Oral Surgery, School of Dentistry, Medical University of Vienna, Sensengasse 2a, 1090 Vienna, Austria.

Received: 7 December 2016 Accepted: 3 May 2017

Published online: 25 May 2017

\section{References}

1. Shibata A, Ibaragi S, Mandai H, Tsumura T, Kishimoto K, Okui T, Hassan NMM, Shimo T, Omori K, Hu G-F, Takashiba S, Suga S, Sasaki A. Synthetic terrein inhibits progression of head and neck cancer by suppressing angiogenin production. Anticancer Res. 2016:36:2161-8.

2. Tsuji T, Sun Y, Kishimoto K, Olson KA, Liu S, Hirukawa S, Hu G-F. Angiogenin is translocated to the nucleus of HeLa cells and is involved in ribosomal RNA transcription and cell proliferation. Cancer Res. 2005;65:1352-60. 
3. Shi H, Han C, Mao Z, Ma L, Gao C. Enhanced angiogenesis in porous collagen-chitosan scaffolds loaded with angiogenin. Tissue Eng Part A. 2008; 14:1775-85.

4. Kim B-S, Kim J-S, Yang S-S, Kim H-W, Lim HJ, Lee J. Angiogenin-loaded fibrin/bone powder composite scaffold for vascularized bone regeneration. Biomater Res. 2015;19:18.

5. Fierro FA, O'Neal AJ, Beegle JR, Chávez MN, Peavy TR, Isseroff RR, Egaña JT. Hypoxic pre-conditioning increases the infiltration of endothelial cells into scaffolds for dermal regeneration pre-seeded with mesenchymal stem cells. Front Cell Dev Biol. 2015:3:68.

6. Pan S-C, Wu L-W, Chen C-L, Shieh S-J, Chiu H-Y. Angiogenin expression in burn blister fluid: implications for its role in burn wound neovascularization. Wound Repair Regen. 2012;20:731-9.

7. Wang $X$, Zhang $Y$, Han C. Angiogenin, an angiogenic factor with potential for tissue engineering applications. Wound Repair Regen. 2014;22:288-9.

8. Sheng J, Xu Z. Three decades of research on angiogenin: a review and perspective. Acta Biochim Biophys Sin (Shanghai). 2016;48:399-410.

9. El Karim IA, Linden GJ, Irwin CR, Lundy FT. Neuropeptides regulate expression of angiogenic growth factors in human dental pulp fibroblasts. $J$ Endod. 2009;35:829-33.

10. Chung CJ, Kim E, Song M, Park J-W, Shin S-J. Effects of two fast-setting calcium-silicate cements on cell viability and angiogenic factor release in human pulp-derived cells. Odontology. 2016;104:143-51.

11. Koutroubakis IE, Xidakis C, Karmiris K, Sfiridaki A, Kandidaki E, Kouroumalis EA. Serum angiogenin in inflammatory bowel disease. Dig Dis Sci. 2004;49:1758-62

12. Oikonomou KA, Kapsoritakis AN, Kapsoritaki Al, Manolakis AC, Tiaka EK Tsiopoulos FD, Tsiompanidis IA, Potamianos SP. Angiogenin, angiopoietin-1 angiopoietin-2, and endostatin serum levels in inflammatory bowel disease. Inflamm Bowel Dis. 2011;17:963-70.

13. Kieran D, Sebastia J, Greenway MJ, King MA, Connaughton D, Concannon CG, Fenner B, Hardiman O, Prehn JHM. Control of motoneuron survival by angiogenin. J Neurosci. 2008;28:14056-61.

14. Hooper LV, Stappenbeck TS, Hong CV, Gordon Jl. Angiogenins: a new class of microbicidal proteins involved in innate immunity. Nat Immunol. 2003;4:269-73.

15. Weremowicz S, Fox EA, Morton CC, Vallee BL. Localization of the human angiogenin gene to chromosome band $14 q 11$, proximal to the T cell receptor alpha/delta locus. Am J Hum Genet. 1990;47:973-81.

16. Shapiro R, Riordan JF, Vallee BL. Characteristic ribonucleolytic activity of human angiogenin. Biochemistry. 1986;25:3527-32.

17. Morelli T, Neiva R, Nevins ML, McGuire MK, Scheyer ET, Oh T-J, Braun TM, Nör JE, Bates D, Giannobile WV. Angiogenic biomarkers and healing of living cellular constructs. J Dent Res. 2011;90:456-62.

18. Weiss $S$, Zimmermann $G$, Pufe $T$, Varoga D, Henle P. The systemic angiogenic response during bone healing. Arch Orthop Trauma Surg. 2009;129:989-97.

19. Kishimoto K, Liu S, Tsuji T, Olson KA, Hu G-F. Endogenous angiogenin in endothelial cells is a general requirement for cell proliferation and angiogenesis. Oncogene. 2005:24:445-56.

20. Jimi S, Ito K, Kohno K, Ono M, Kuwano M, Itagaki Y, Ishikawa H. Modulation by bovine angiogenin of tubular morphogenesis and expression of plasminogen activator in bovine endothelial cells. Biochem Biophys Res Commun. 1995:211:476-83.

21. Xu Z, Tsuji T, Riordan JF, Hu G. The nuclear function of angiogenin in endothelial cells is related to rRNA production. Biochem Biophys Res Commun. 2002;294:287-92

22. Gao X, Xu Z. Mechanisms of action of angiogenin. Acta Biochim Biophys Sin (Shanghai). 2008:40:619-24

23. Li S, Hu G-F. Emerging role of angiogenin in stress response and cell survival under adverse conditions. J Cell Physiol. 2012;227:2822-6.

24. Müller H-D, Cvikl B, Janjić K, Nürnberger S, Moritz A, Gruber R, Agis H. Effects of prolyl hydroxylase inhibitor L-mimosine on dental pulp in the presence of advanced glycation end products. J Endod. 2015;41:1852-61.

25. Kuchler U, Keibl C, Fügl A, Schwarze UY, Tangl S, Agis H, Gruber R. Dimethyloxalylglycine lyophilized onto bone substitutes increase vessel area in rat calvarial defects. Clin Oral Implants Res. 2015;26:485-91.

26. Hadjipanayi E, Schilling AF. Hypoxia-based strategies for angiogenic induction: the dawn of a new era for ischemia therapy and tissue regeneration. Organogenesis. 2013;9:261-72.

27. Beegle J, Lakatos K, Kalomoiris S, Stewart H, Isseroff RR, Nolta JA, Fierro FA. Hypoxic preconditioning of mesenchymal stromal cells induces metabolic changes, enhances survival, and promotes cell retention in vivo. Stem Cells. 2015:33:1818-28.

28. Nouri F, Salehinejad P, Nematollahi-Mahani SN, Kamarul T, Zarrindast MR, Sharifi AM. Deferoxamine preconditioning of neural-like cells derived from human Wharton's jelly mesenchymal stem cells as a strategy to promote their tolerance and therapeutic potential: an in vitro study. Cell Mol Neurobiol. 2016;36:689-700.

29. Liu X-B, Wang J-A, Ji X-Y, YU SP, Wei L. Preconditioning of bone marrow mesenchymal stem cells by prolyl hydroxylase inhibition enhances cell survival and angiogenesis in vitro and after transplantation into the ischemic heart of rats. Stem Cell Res Ther. 2014:5:111.

30. Jiang L, Peng W-W, Li L-F, Du R, Wu T-T, Zhou Z-J, Zhao J-J, Yang Y, Qu D-L, Zhu Y-Q. Effects of deferoxamine on the repair ability of dental pulp cells in vitro. J Endod. 2014;40:1100-4.

31. Nakamura M, Yamabe H, Osawa H, Nakamura N, Shimada M, Kumasaka R, Murakami R, Fujita T, Osanai T, Okumura K. Hypoxic conditions stimulate the production of angiogenin and vascular endothelial growth factor by human renal proximal tubular epithelial cells in culture. Nephrol Dial Transplant. 2006;21:1489-95.

32. Lai K, Luo C, Zhang X, Ye P, Zhang Y, He J, Yao K. Regulation of angiogenin expression and epithelial-mesenchymal transition by HIF-1a signaling in hypoxic retinal pigment epithelial cells. Biochim Biophys Acta. 1862;2016:1594-607.

33. Müller H-D, Cvikl B, Gruber R, Watzek G, Agis H. Prolyl hydroxylase inhibitors increase the production of vascular endothelial growth factor in dental pulp-derived cells. J Endod. 2012;38:1498-503.

34. Gruber R, Kandler B, Agis H, Fischer MB, Watzek G. Bone cell responsiveness to growth and differentiation factors under hypoxia in vitro. Int J Oral Maxillofac Implants. 2008;23:417-26.

35. Trimmel K, Cvikl B, Müller H-D, Nürnberger S, Gruber R, Moritz A, Agis H. Lmimosine increases the production of vascular endothelial growth factor in human tooth slice organ culture model. Int Endod J. 2015;48:252-60.

36. Iohara K, Zheng L, Ito M, Tomokiyo A, Matsushita K, Nakashima M. Side population cells isolated from porcine dental pulp tissue with self-renewal and multipotency for dentinogenesis, chondrogenesis, adipogenesis, and neurogenesis. Stem Cells. 2006:24:2493-503.

37. Laino G, d Aquino R, Graziano A, Lanza V, Carinci F, Naro F, Pirozzi G, Papaccio G. A new population of human adult dental pulp stem cells: a useful source of living autologous fibrous bone tissue (LAB). J Bone Miner Res. 2005;20:1394-402.

38. Mirabet V, Solves P, Miñana MD, Encabo A, Carbonell-Uberos F, Blanquer A, Roig R. Human platelet lysate enhances the proliferative activity of cultured human fibroblast-like cells from different tissues. Cell Tissue Bank. 2008:9:1-10.

39. Suchanek J, Soukup T, Visek B, Ivancakova R, Kucerova L, Mokry J. Dental pulp stem cells and their characterization. Biomed Pap Med Fac Univ Palacky Olomouc Czech Repub. 2009;153:31-5

40. Pérard M, Le Clerc J, Watrin T, Meary F, Pérez F, Tricot-Doleux S, PellenMussi P. Spheroid model study comparing the biocompatibility of Biodentine and MTA. J Mater Sci Mater Med. 2013:24:1527-34.

41. Hsiao ST, Lokmic Z, Peshavariya H, Abberton KM, Dusting GJ, Lim SY, Dilley RJ. Hypoxic conditioning enhances the angiogenic paracrine activity of human adipose-derived stem cells. Stem Cells Dev. 2013;22:1614-23.

42. Wakayama $\mathrm{H}$, Hashimoto $\mathrm{N}$, Matsushita $\mathrm{Y}$, Matsubara $\mathrm{K}$, Yamamoto $\mathrm{N}$ Hasegawa Y, Ueda M, Yamamoto A. Factors secreted from dental pulp stem cells show multifaceted benefits for treating acute lung injury in mice. Cytotherapy. 2015:17:1119-29.

43. Fujio M, Xing Z, Sharabi N, Xue Y, Yamamoto A, Hibi H, Ueda M, Fristad I, Mustafa K. Conditioned media from hypoxic-cultured human dental pulp cells promotes bone healing during distraction osteogenesis. J Tissue Eng Regen Med. 2015.

44. Werle SB, Chagastelles P, Pranke P, Casagrande L. The effects of hypoxia on in vitro culture of dental-derived stem cells. Arch Oral Biol. 2016;68:13-20.

45. Yamaguchi S, Shibata R, Yamamoto N, Nishikawa M, Hibi H, Tanigawa T, Ueda M, Murohara T, Yamamoto A. Dental pulp-derived stem cell conditioned medium reduces cardiac injury following ischemia-reperfusion. Sci Rep. 2015:5:16295.

46. Cho Y-H, Park H, Cho E-S, Kim W-J, Kang B-S, Park B-Y, Kim Y-J, Lee Y-I, Chang S-I, Park K. A novel way of therapeutic angiogenesis using an adenoassociated virus-mediated angiogenin gene transfer. Exp Mol Med. 2007;39:412-8 